\title{
Spectral density and magnetic susceptibility for the asymmetric degenerate Anderson model
}

\author{
F. C. Zhang \\ School of Physics and Astronomy, University of Minnesota, Minneapolis, Minnesota 55455 \\ T. K. Lee \\ Department of Physics, Virginia Polytechnic Institute and State University, \\ Blacksburg, Virginia 24061 \\ (Received 26 April 1984)
}

\begin{abstract}
With the use of a new diagrammatic formulation, two coupled integral equations for the self-energy functions of the $f$ hole and $f$ particle in the asymmetric degenerate Anderson model are solved numerically. All the diagrams are included in the equations except the cross terms (or the vertex correction). The results for the spectral density function and the magnetic susceptibility show the scaling property described by the renormalization-group theory.
\end{abstract}

We present the numerical results of the spectral density function for the asymmetric degenerate Anderson impurity model. ${ }^{1,2}$ Using a new diagrammatic formulation, ${ }^{3}$ we have solved two coupled integral equations $s^{4,5}$ for the $f$-hole and $f$-particle self-energy functions. The scaling property of the Anderson model described by the renormalization-group ${ }^{6}$ theory (RGT) and scaling theory ${ }^{7}$ is demonstrated in our result. The temperature-dependent magnetic susceptibility is found to be in excellent agreement with the universal curve obtained by RGT in the Kondo regime.

This is the first time that a diagrammatic method is shown to give accurate results for the Anderson impurity model in the Kondo regime. This method and its quantitative results of the spectral density may be generalized and used to study systems with many Anderson impurities, such as fluctuating valence compounds, the Kondo lattice systems, and the heavy-fermion superconductors. ${ }^{8}$ On the other hand, it is extremely difficult to generalize the $\mathrm{RGT}^{6,9}$ and the method of Bethe ansatz. ${ }^{10}$

More than ten years ago Keiter and Kimball ${ }^{5}$ presented a Goldstone diagrammatic technique for the Anderson model with a single impurity. However, there are practical difficulties in the Keiter and Kimball theory. Using the BrillouinWigner equations, they must adopt a very complicated regularization procedure ${ }^{5,11}$ to avoid accidentally vanishing energy denominators. To overcome this difficulty, recently we have revised the approach taken by Keiter and Kimball. ${ }^{3}$ Simultaneously, several similar approaches also have been presented. ${ }^{12-14}$ But the final equations of all these approaches completely agree with each other. Gunnarsson and Shönhammer ${ }^{15}$ have calculated the spectral density at zero temperature in the large- $N$ limit.

We consider the asymmetric Anderson model ${ }^{3,4}$ in the limit of infinitely large Coulomb interaction $U$. In Ref. 3, we showed that the partition function is given by

$$
\begin{aligned}
\frac{Z}{\left(Z_{0}\right)_{c}}= & \oint \frac{d z}{2 \pi i} \frac{e^{-\beta z}}{z-S(z)} \\
& +\sum_{m} \oint \frac{d z}{2 \pi i} \frac{e^{-\beta z}}{z-\epsilon_{f m}-T_{m}\left(z-\epsilon_{f m}\right)}
\end{aligned}
$$

where $\left(Z_{0}\right)_{c}$ is the partition function for the noninteracting conduction electrons, $\boldsymbol{\epsilon}_{f m}$ the energy separation between the configurations $|m\rangle\left(4 f^{n-1}, J, m\right)$ and $|0\rangle\left(4 f^{n}, J=0\right)$, and the contour circles all the poles. There are $N=2 J+1$ degenerate configurations $|m\rangle . S(z)$ and $T_{m}(z)$ are the selfenergy function of configurations $|0\rangle$ ( $f$ hole) and $|m\rangle(f$ particle), respectively. They are constructed according to the Goldstone diagrammatic rule given by Keiter and Kimball. ${ }^{5}$ Since $[z-S(z)]^{-1}$ and $\left[z-\epsilon_{f m}-T_{m}\left(z-\epsilon_{f m}\right)\right]^{-1}$ are analytic everywhere except on the real axis, we shall be only concerned with $z=x-i \delta$, where $\delta=0^{+}$. The self-energy functions $S$ and $T_{m}$ can be calculated perturbatively in series of the hybridization energy $\Delta=\Sigma_{k \sigma}\left|V_{k \sigma m}\right|^{2} \delta\left(\epsilon_{k \sigma}-\omega\right)$.

A better way is to use $N^{-1}$ as a small parameter and make a $1 / N$ expansion. ${ }^{16}$ In Ref. 4 , we have calculated the ground-state properties by using a $1 / N$ series of self-energy functions $S$ and $T$. The series quickly converges in the mixed-valence regime where $\epsilon_{f}$ is not very negative (the Fermi energy is set to be zero). As the $1 / N$ expansion is a perturbative method of summing up diagrams in $1 / N$ order. It is well known that in the Kondo regime the infrared divergence cannot be eliminated by this kind of perturbative approach. The approach described below overcomes this difficulty.

If we neglect the vertex correction (or the cross terms ${ }^{4}$ ) in the absence of a magnetic field, the self-energy functions $S$ and $T_{m}$ satisfy the following integral equations: $:^{4,5}$

$$
S(z)=\int_{-D}^{D} d \epsilon \frac{f(\epsilon)}{z+\epsilon-\epsilon_{f}-T_{m}\left(z+\epsilon-\epsilon_{f}\right)}
$$

and

$$
T_{m}(z)=\frac{1}{N} \int_{-D}^{D} d \epsilon \frac{f \epsilon}{z+\epsilon+\epsilon_{f}-S\left(z+\epsilon+\epsilon_{f}\right)}
$$

where $2 D$ is the bandwidth and a flat density of states has been assumed. For convenience, we take units $N \Delta=1$ in this paper.

We have solved the integral equations (1) and (2) numerically and then use the solutions to calculate the magnetic susceptibility $\chi$ and the spectral density function $R_{m}(\omega)=\operatorname{Im} G_{m m}(\omega-i \delta) / \pi$. Details of the numerical methods will be given in a future publication. ${ }^{17}$ 
TABLE I. Numerical values associated with curves A, B, C, and D of Fig. 1. Curves B, C, and D are also in Fig. 2, and curve A in Fig. 3. We have taken the unit $N \Delta=1 . \epsilon_{f}^{*}, \tilde{\epsilon}_{f}$, and $\tilde{\epsilon}_{f}^{\prime}$ are calculated by Eqs. (3)-(5). $\quad n_{f}$ is the occupation number. $\chi$ is in unit of $g^{2} \mu_{B}^{2} J(J+1) / 3$.

\begin{tabular}{lccccccc}
\hline \hline Curve & $N$ & $D$ & $\epsilon_{f}$ & $\epsilon_{f}^{*}$ & $\tilde{\epsilon}_{f}\left[\right.$ or $\left.\tilde{\epsilon}_{f}^{\prime}\right]$ & $\chi$ & $n_{f}$ \\
\hline A & 2 & 100 & -7.5 & -5.20 & -6.1 & 230 & 0.906 \\
B & 6 & 32.9 & -5 & -2.09 & -3.10 & 9.14 & 0.856 \\
C & 6 & 32.9 & -2.5 & 0.41 & 0.736 & 0.865 & 0.552 \\
D & 6 & 32.9 & 0 & 2.91 & 2.29 & 0.148 & 0.292 \\
\hline \hline
\end{tabular}

At zero temperature the integral equations can be changed to differential equations. One of the differential equations can be integrated analytically. Taking appropriate boundary conditions, we obtain an integration constant

$$
\epsilon_{f}^{*}=\epsilon_{f}+\left(1-N^{-1}\right) \ln D \text {. }
$$

For $N=2, \epsilon_{f}^{*}$ is exactly the scaling invariant described by Haldane $^{7}$ in his scaling theory for $J=\frac{1}{2}$. Just like the prediction from $\mathrm{RGT}^{6}$ and scaling theory, ${ }^{7}$ depending on the value of $\epsilon_{f}^{*}$ the solutions of the integral equations can be classified into two different regimes: the mixed-valence regime where $\epsilon_{f}^{*} \geq-1$ (Ref. 18) and the Kondo regime where $\epsilon_{f}^{*} \leq-1$.

In the mixed-valence regime, there is only one solution $E_{0}$ for the equation $x=\operatorname{Re} S(x-i \delta)$. At finite temperature there is also only one solution $E_{m}$ for $\operatorname{Re} T_{m}\left(E_{m}-i \delta\right)=E_{m}$. $E_{0}$ is always less than $E_{m}+\epsilon_{f}$; therefore, the ground state is a singlet. In the last two rows of Table I, where $\epsilon_{f}^{*}$ are positive, we have listed the values of $E_{m}+\epsilon_{f}-E_{0}=\tilde{\epsilon}_{f}$. It is easy to show that they are in good agreement ${ }^{19}$ with the scaling equation

$$
\tilde{\epsilon}_{f}+\left(1-N^{-1}\right) \ln \tilde{\epsilon}_{f}=\epsilon_{f}^{*} \text {. }
$$

According to $\mathrm{RGT},{ }^{6}$ at a temperature $\tilde{T}=\tilde{\boldsymbol{\epsilon}}_{f} / \alpha$ (where $\alpha$ is a constant of order one) the system changes from a free orbital regime where susceptibility is given by

$$
T \chi /\left(g^{2} \mu_{B}^{2}\right)=\frac{J(J+1)}{3} \frac{N}{N+1}
$$

to the frozen impurity regime where $T \chi=0$. This kind of temperature dependence of the susceptibility is shown in curves $\mathrm{C}$ and $\mathrm{D}$ in Fig. 1. Values of $N, \epsilon_{f}, D$, etc., for

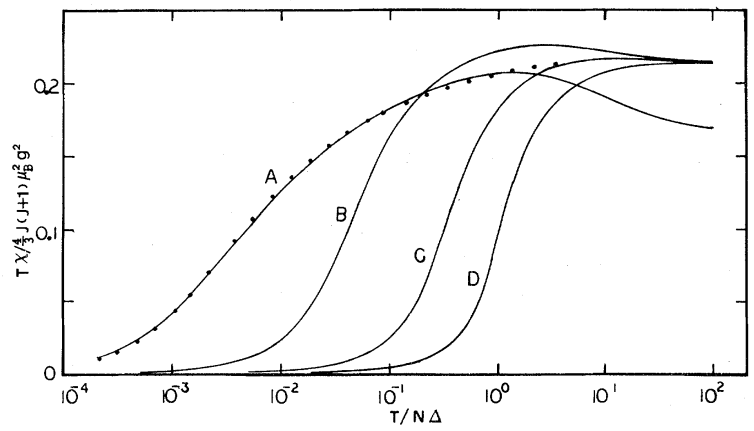

FIG. 1. A semilogarithmic plot of $\left[3 \chi T / 4 J(J+1) \mu_{B}^{2} g^{2}\right]$ vs $T / N \Delta$. The dots are the universal curve for symmetric Anderson model by RGT (Ref. 6). See Table I for numerical values. curves $\mathrm{C}$ and $\mathrm{D}$ are listed in Table $\mathrm{I}$. In this regime the ground-state energy and the magnetic susceptibility at zero temperature obtained by solving the integral equations are in excellent agreement with the result of the $1 / N$ expansion method. ${ }^{4}$

In Fig. 2 the spectral density function $R_{m}(\omega)$ for curves C and $\mathrm{D}$ shows only one peak at $\omega=\tilde{\epsilon}_{f}$, which is always positive. $^{20}$ The peak is rather symmetrical and Lorentzian-like. The half-width is very close to the value of $\operatorname{Im} T_{m}\left(E_{m}-i \delta\right) \cong\left(1-n_{f}\right) \pi / N ; n_{f}$ is the $f$-electron occupation number. The height is only a little less than $N / \pi^{2}$.

There is a very very small peak at $\omega=0$ in curves $C$ and $\mathrm{D}$ of Fig. 2. This peak occurred due to the approximation of neglecting the cross terms (or vertex corrections). Details of this analysis will be given elsewhere. ${ }^{17}$ The leading cross term, calculated in Ref. 4 , is shown to be negligible for large $N$ and large bandwidth. Therefore, the physical quantities calculated from the integral equations should be very accurate in spite of the approximation of neglecting the cross terms.

At $T=0 \mathrm{~K}$, the spectral density $R_{m}(\omega)$ at $\omega=0$ should satisfy the Friedel sum rule. ${ }^{21}$ Curves $C$ and D in Fig. 2 disagree with the sum rule. If we ignore the peak at $\omega=0$ and just assume a Lorentzian centered at $\tilde{\epsilon}_{f}$ having width $\left(1-n_{f}\right) \pi / N$, then fairly good agreement with the sum rule at $\omega=0$ is obtained.

For very negative $\epsilon_{f}$, the system is in the Kondo regime. At moderate temperature, both functions $x-\operatorname{Re} S(x-i \delta)$ and $x-\epsilon_{f}-\operatorname{Re} T_{m}\left(x-\epsilon_{f}-i \delta\right)$ have only one root $E_{02}$ and $E_{m}+\epsilon_{f}$, respectively, and $E_{02}>E_{m}+\epsilon_{f}$. The physics is controlled by the magnetic states $|m\rangle$. The numerical results show that the effective $\epsilon_{f}$, i.e., $\tilde{\epsilon}_{f}^{\prime}=E_{m}+\epsilon_{f}-E_{02}$,

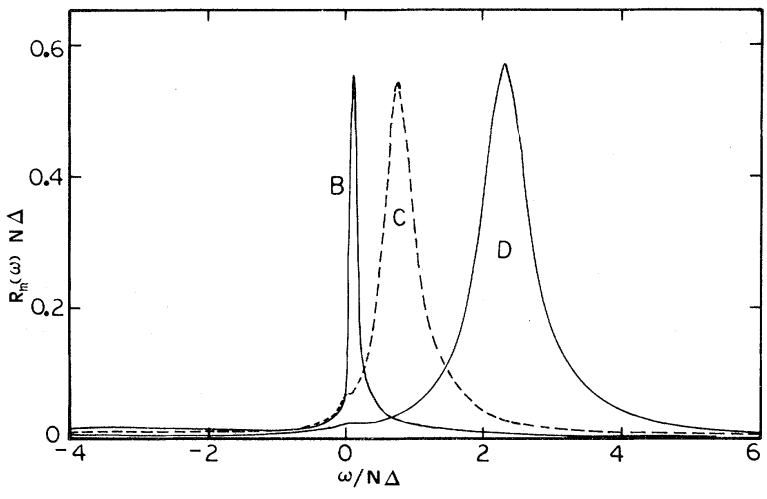

FIG. 2. Spectral density $R_{m}(\omega) N \Delta$ for $N=6, D / N \Delta=32.9$. See Table I for numerical values. 
satisfies the scaling equation ${ }^{6}$

$$
-\tilde{\epsilon}_{f}^{\prime}-\left(1-N^{-1}\right) \ln -\tilde{\epsilon}_{f}^{\prime}=-\epsilon_{f}^{*} .
$$

The values of $\tilde{\boldsymbol{\epsilon}}_{f}^{\prime}$ for curves A and B in Fig. 1 are listed in Table I. At a temperature $\tilde{T}_{\text {eff }}=\left|\tilde{\epsilon}_{f}^{\prime}\right| / \alpha$, the system changes from a free orbital regime to the local moment regime where $T \chi /\left(g^{2} \mu_{B}^{2}\right)$ approaches $J(J+1) / 3$, as shown in Fig. 1.

As the temperature decreases below the Kondo temperature, the function $x-\operatorname{Re} S(x-i \delta)$ has two more roots $E_{0}$ and $E_{01}$, where $E_{0}<E_{m}+\epsilon_{f}<E_{01}<E_{02}$. Since $E_{0}$ is less than $E_{m}+\epsilon_{f}$, the ground state is the singlet. The moment has now been quenched. The Kondo temperature $T_{K}$ is proportional to $T_{K} \sim \exp \left(\epsilon_{f}^{*}\right)=D^{\left(1-N^{-l}\right)} e^{\epsilon_{f}}$.

In Fig. 1 curves $A$ and $B$ both show the transition from local moment regime to the frozen impurity regime at Kondo temperature, as described by RGT. ${ }^{6}$ The parameters for curves $\mathrm{A}$ and $\mathrm{B}$ are listed in Table I. Curve A shows an excellent agreement with the universal curve ${ }^{6}$ (dotted line). The Kondo temperature $T_{K}^{\prime}$ of the universal curve is determined by requiring $T_{K}^{\prime} \chi=0.07$; therefore, $T_{K}^{\prime}=0.002$ for curve A.

In the Kondo regime $R_{m}(\omega)$ has two peaks. In Fig. 3, $R_{m}(\omega)$ is plotted for three different temperatures: (A) $T=6 \times 10^{-4},\left(\mathrm{~A}^{\prime}\right) T=10^{-2}$, and ( $\left.\mathrm{A}^{\prime \prime}\right) T=0.3$. Values of $N, \epsilon_{f}$, and $D$ are given in Table I, curve A. The curve B in Fig. 2 and the inset in Fig. 3 show a broad peak at $\omega=E_{m}+\epsilon_{f}-E_{02}=\tilde{\epsilon}_{f}^{\prime}<0$. Its half-width is about $\operatorname{Im} S\left(E_{02}-i \delta\right)=\pi$ and the height is $n_{f} / N \pi^{2}$. The presence of the very sharp and asymmetric peak near $\omega=0$ is the characteristic of Kondo behavior predicted from scaling theory. This peak is centered at $\omega=E_{m}+\epsilon_{f}-E_{0}$, just as the peak at $\omega=\tilde{\epsilon}_{f}$ for curves $C$ and $D$ in the mixed-valence regime. When the peak gets close to $\omega=0$, its width gets smaller and it becomes asymmetric. The asymmetry is due to the particle-hole asymmetry of the Hamiltonian. The half-width of the peak is approximately $T_{K} \pi / N$. The line shape of this peak is essentially determined by the imaginary part of $T_{m}(x-i \delta)$ for $x$ near $E_{m}$.

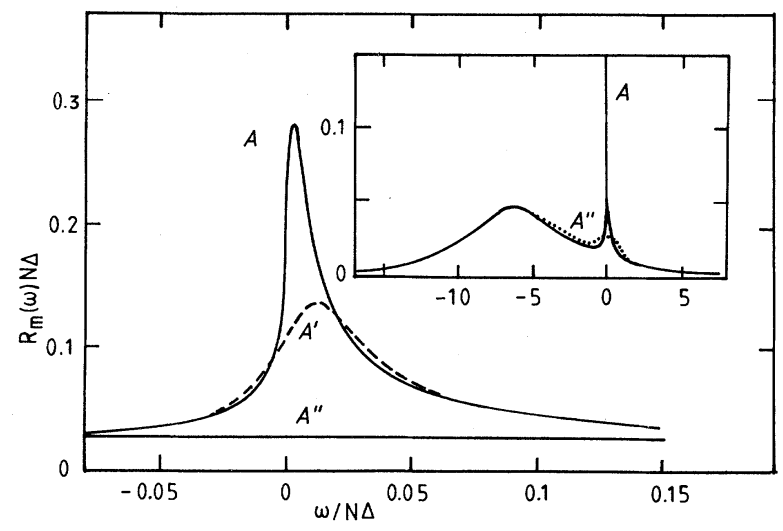

FIG. 3. Central peak of the spectral density $R_{m}(\omega) N \Delta$ for $N=2$, $D / N \Delta=100, \epsilon_{f} / N \Delta=-7.5$. Curves $\mathrm{A}, \mathrm{A}^{\prime}$, and $\mathrm{A}^{\prime \prime}$ are for $T / N \Delta=6 \times 10^{-4}, 10^{-2}$, and 0.3 , respectively. The inset shows $R_{m}(\omega)$ in a wider range.

Because the peak at $\omega=E_{m}+\epsilon_{f}-E_{0}$ is very close to $\omega=0$, the effect of neglecting cross terms exhibited by the very small unphysical peak at $\omega=0$ in curves $C$ and $D$ of Fig. 2 is not found here. In fact the prediction of Friedel sum rule, $R_{m}(0)=0.11$ for curve $\mathrm{B}$ in Fig. 2 and $R_{m}(0)=0.2$ for curve $\mathrm{A}$ in Fig. 3 , agree very well with the numerical results $R_{m}(0)=0.11$ and 0.21 , respectively.

In summary, we have numerically solved two coupled integral equations for the $f$-hole and $f$-particle self-energy functions $S(x-i \delta)$ and $T_{m}(x-i \delta)$, respectively. The scaling property of the Anderson model is clearly exhibited in our result of magnetic susceptibility and the spectral density function.

One of us (F.C.Z.) would like to thank Professor C. E. Campbell and Professor O. T. Valls for their encouragement. This work was supported in part by the Micorelectronic Information Science Center of University of Minnesota.
${ }^{1}$ Valence Fluctuation in Solids, edited by L. M. Falicov, W. Hanke, and M. B. Maple (North-Holland, Amsterdam, 1981).

2 Valence Instabilities, edited by P. Wachter and H. Boppart (NorthHolland, Amsterdam, 1982).

${ }^{3}$ T. K. Lee and F. C. Zhang, J. Appl. Phys. 55, 1936 (1984); F. C. Zhang, thesis (unpublished).

${ }^{4}$ F. C. Zhang and T. K. Lee, Phys. Rev. B 28, 33 (1983).

${ }^{5}$ H. Keiter and J. C. Kimball, Int. J. Magn. 1, 233 (1971)

${ }^{6} \mathrm{H}$. R. Krishna-murthy, J. W. Wilkins, and K. G. Wilson, Phys. Rev. B 21, 1044 (1980).

${ }^{7}$ F. D. M. Haldane, Phys. Rev. Lett. 40, 416 (1978); 40, 911(E) (1978).

${ }^{8}$ H. R. Ott, H. Rudigier, Z. Fisk, and J. L. Smith, Phys. Rev. Lett 50, 1595 (1983)

${ }^{9}$ L. N. Oliveira and J. W. Wilkins, Phys. Rev. Lett. 47, 1533 (1981).

10P. Schlottmann, Z. Phys. B 49, 109 (1982), and references therein.
${ }^{11}$ G. Czycholl, H. Keiter, and E. Niebur, in Ref. 2, p. 45.

12Y. Kuramoto (unpublished); J. Magn. Magn. Mater. 31-34, 463 (1983).

13P. Coleman, Phys. Rev. B 29, 3035 (1984). The formalism is different from Refs. 3 and 14.

${ }^{14}$ N. Grewe, Z. Phys. B 53, 271 (1983).

${ }^{15} \mathrm{O}$. Gunnarsson and K. Shönhammer, Phys. Rev. B 28, 4315 (1983).

${ }^{16} \mathrm{~T}$. V. Ramakrishnan, in Ref. 1, p. 13.

${ }^{17}$ F. C. Zhang and T. K. Lee (unpublished).

${ }^{18}-1$ is obtained for very large $N$.

${ }^{19}$ In fact, we should have $\tilde{\boldsymbol{\epsilon}}_{f}+[1-(1 / N)] \ln \tilde{\boldsymbol{\epsilon}}_{f} / \gamma=\epsilon_{f}^{*}$, where $\gamma=1.088$. But $\gamma$ approaches 1 when we increase the bandwidth.

${ }^{20}$ Curves in Fig. 2 are calculated at temperatures that all the physical quantities become unchanged when temperature varies.

${ }^{21}$ D. C. Langreth, Phys. Rev. 150, 516 (1966). 